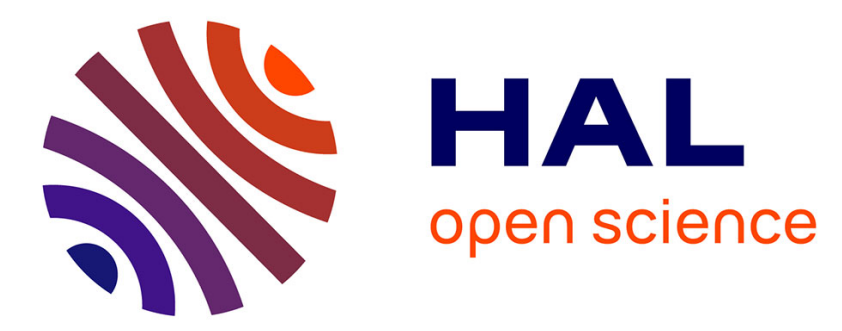

\title{
Characterization of capacitive MEMS resonators via nonlinear open-loop frequency responses
}

Alexis Brenes, Jérôme Juillard, Laurent Bourgois, Filipe Vinci dos Santos

\section{To cite this version:}

Alexis Brenes, Jérôme Juillard, Laurent Bourgois, Filipe Vinci dos Santos. Characterization of capacitive MEMS resonators via nonlinear open-loop frequency responses. 2015 Symposium on Design, Test, Integration and Packaging of MEMS/MOEMS (DTIP), Apr 2015, Montpellier, France. pp.1-6, 10.1109/DTIP.2015.7161022 . hal-01235634

\section{HAL Id: hal-01235634}

https://hal-centralesupelec.archives-ouvertes.fr/hal-01235634

Submitted on 4 Dec 2015

HAL is a multi-disciplinary open access archive for the deposit and dissemination of scientific research documents, whether they are published or not. The documents may come from teaching and research institutions in France or abroad, or from public or private research centers.
L'archive ouverte pluridisciplinaire $\mathbf{H A L}$, est destinée au dépôt et à la diffusion de documents scientifiques de niveau recherche, publiés ou non, émanant des établissements d'enseignement et de recherche français ou étrangers, des laboratoires publics ou privés. 


\section{Characterization of capacitive MEMS resonators via nonlinear open-loop frequency responses}

\author{
A. Brenes \\ THALES Avionics \\ Valence, France \\ alexis.brenes@supelec.fr
}

\author{
J. Juillard ${ }^{1}$, L. Bourgois ${ }^{1}$ and F. Vinci dos Santos ${ }^{2}$ \\ E3S ${ }^{1}$ and THALES Chair ${ }^{2}$ \\ Ecole Supérieure d'Electricité (Supélec) \\ Gif-sur-Yvette, France
}

\begin{abstract}
The aim of this paper is to demonstrate robust parameter extraction from the frequency response of MEMS devices exhibiting pronounced nonlinearities. We describe a general methodology, based on parameter extraction from nonlinear large-signal frequency response, whose accuracy appears to be limited in practice only by noise and by the mathematical model of the structure under consideration. We illustrate this by two complete case studies of capacitive MEMS resonators driven to large-amplitude regimes. Theoretical analysis, simulations and experimental measurements strongly support our conclusions.
\end{abstract}

Keywords- Electrostatic nonlinearity, open-loop frequency response, clamped-clamped resonator, parallel-plate resonator, MEMS

\section{INTRODUCTION}

In this paper, we give evidence of the advantages of a general characterization method based on parameter extraction from a nonlinear resonant frequency response. We adapt our method to capacitive MEMS driven to large amplitude regimes and show that our parameter extraction procedure succeeds in obtaining accurate values.

Commonly, the characterization of a resonator consists in estimating its natural frequency and Q-factor, either via openloop frequency response curves [1], or exponential decay time measurements [2]. To carry out this characterization, all the forces applied on the resonator are considered to give linear responses, or are linearized assuming that the resonator operates in small amplitude oscillation regimes. Under these assumptions, measurements are hampered by low SNR. This obstacle gives impulse to the investigation of frequency responses in large amplitude regimes, in which the nonlinear behavior of the resonator cannot be ignored.

In nonlinear regimes, open-loop frequency responses are distorted so that the symmetry of amplitude resonance curves around the natural frequency is broken, sometimes resulting in the emergence of hysteresis cycles. A recent study has demonstrated Q-factor estimation from a nonlinear frequency response [3], via precise measurements at low amplitudes (hence at low SNR) and the knowledge of the maximal amplitude on the frequency response. However, both of them are difficult to measure in practice. Another study has demonstrated the efficacy of nonlinear least-squares fitting for parameter estimation of piezoelectric MEMS resonators from a nonlinear frequency response exhibiting hysteresis [4]. This method is also experimentally demanding because it requires numerous measurements around the upper bifurcation point, a difficult endeavor since this point is, by definition, unstable in open loop regimes. These impediments gave impulse to our search for a new approach.

Our study is focalized on capacitive MEMS frequency responses. These responses are known to be distorted by the parasitic current effects in linear [5] and nonlinear [6] regimes. However, a few methods avoid this problem, whether via subharmonic sine-wave actuation $[7,8]$ or via de-embedded pulsed-mode actuation [9]. In this paper, we will assume that the nonlinear frequency response is obtained without significant parasitic current effects.

This paper is organized as follows: in Section II, the general background required to perform parameter extraction of a nonlinear response is presented. This general model has already been disclosed, and was adapted to practical uses under restrictive assumptions [10]. In Section III, we show how this scheme can be used for the characterization of plane-capacitance and clamped-clamped MEMS resonators. Section IV illustrates parameter extraction on different synthetic (i.e. simulated) data sets corresponding to the presented models. Finally, Section V validates the method with experimental measurements.

\section{DYNAMIC BEHAVIOR OF MEMS RESONATORS}

MEMS resonators exhibit a large variety of nonlinearities, from hardening to softening effects [11]. For instance, the behavior of a resonator under sine-wave actuation may be described by:

$$
\frac{d^{2} x}{d t^{2}}+\frac{\omega_{0}}{Q} \frac{d x}{d t}+\omega_{0}^{2} x=G^{\theta}(x)+F^{\theta}(x) \sin (\omega t+\varphi)
$$

where $x$ is the normalized displacement of the resonator, $\omega_{0}$ its natural frequency, $Q$ its Q-factor and $F^{\theta}$ and $G^{\theta}$ normalized forces depending on a vector $\theta$ of parameters. For the sake of simplicity, we will assume that the static displacement of the resonator can be neglected. The equations may be adapted to take into account this static displacement. Calculations are tedious but straightforward. Then, assuming that $F^{\theta}$ and $G^{\theta}$ only exhibit static memory-less nonlinearities and $x(t)=A \sin \omega t$ (where $0<A<1), F^{\theta}(x(t))$ and $G^{\theta}(x(t))$ may be expressed: 


$$
\begin{aligned}
& F^{\theta}(x(t))=\sum_{k=0}^{\infty} F_{2 k+1}^{\theta}(A) \sin ((2 k+1) \omega t)+F_{2 k}^{\theta}(A) \cos (2 k \omega t) \\
& G^{\theta}(x(t))=\sum_{k=0}^{\infty} G_{2 k+1}^{\theta}(A) \sin ((2 k+1) \omega t)+G_{2 k}^{\theta}(A) \cos (2 k \omega t)
\end{aligned}
$$

Equation (1) may be analyzed with the method of harmonic balance. Considering (2), this method yields:

$$
\begin{aligned}
& \left(\omega_{0}^{2}-\omega^{2}\right) A-G_{1}^{\theta}(A)=\tau_{-}^{\theta}(A) \cos \varphi \\
& \frac{\omega \omega_{0} A}{Q}=\tau_{+}^{\theta}(A) \sin \varphi
\end{aligned}
$$

where $\tau_{ \pm}^{\theta}(A)=F_{0}^{\theta}(A) \pm \frac{F_{2}^{\theta}(A)}{2}$.

Finally:

$$
\begin{aligned}
& h\left(A, \omega, \omega_{0}, Q, \theta\right)=1- \\
& A^{2}\left[\frac{\left[\left(\omega_{0}^{2}-\omega^{2}\right)-\frac{G_{1}^{\theta}(A)}{A}\right]^{2}}{\tau_{-}^{\theta}(A)^{2}}+\frac{\omega^{2} \omega_{0}^{2}}{Q^{2} \tau_{+}^{\theta}(A)^{2}}\right]=0
\end{aligned}
$$

Equation (4) models the general case. Next, we will cast (4) into formulations suited to various types of devices.

\section{APPLICATION TO MEMS RESONATORS}

In this section, we derive from (4) several formulations adapted to specific MEMS applications. Without loss of generality, we will assume that the oscillation of the resonator gives rise to an output signal of amplitude $V=g A$ where $g$ is an a priori unknown gain.

\section{A. MEMS resonator subject to a cubic nonlinear force}

Assuming a cubic nonlinearity with cubic stiffness $\gamma$ and a linear force $f$, the equation of motion (1) becomes:

$$
\frac{d^{2} x}{d t^{2}}+\frac{\omega_{0}}{Q} \frac{d x}{d t}+\omega_{0}^{2} x=-\gamma \omega_{0}^{2} x^{3}+f \sin (\omega t+\varphi)
$$

Finally, $h$ may be written:

$$
\begin{aligned}
& h\left(V, \omega, g, \omega_{0}, Q, \gamma, f\right)= \\
& 1-\frac{V^{2}}{g^{2} f^{2}}\left[\left(\omega_{0}^{2}-\omega^{2}+\frac{3}{4} \gamma \omega_{0}^{2} \frac{V^{2}}{g^{2}}\right)^{2}+\frac{\omega^{2} \omega_{0}^{2}}{Q^{2}}\right]
\end{aligned}
$$

This expression is suitable for the characterization of MEMS resonator via optical measurements where no electrostatic force is applied. However, it requires the knowledge of the actuation force $f$ or the gain $g$ since $f, g$, and $\gamma$ may not be independently identified in (5).
When the resonator is actuated via an AC voltage, more complex expressions must be considered. In the following examples, we will focus on capacitive MEMS resonators.

\section{B. Electrostatic softening of a capacitive MEMS under the plane-capacitance approximation}

In small-displacement nonlinear regimes, the capacitance of the MEMS resonator may sometimes be approximated by a time-varying plane capacitance. Under this approximation, for a one-sided capacitive MEMS, the equation of motion becomes:

$$
\frac{d^{2} x}{d t^{2}}+\frac{\omega_{0}}{Q} \frac{d x}{d t}+\omega_{0}^{2} x=F(V)
$$

with

$$
F(V) \approx \xi_{p} g^{2} \frac{\left(1+2 V_{0} \sin (\omega t+\varphi)\right)}{(g-V \sin \omega t)^{2}}
$$

where $\xi_{p}=\eta \omega_{0}^{2}, \quad V_{0}=V_{a c t} / V_{b}<<1$ where $V_{a c t}$ is the actuation voltage, $V_{b}$ the bias voltage, $\eta=\varepsilon S V_{b}^{2} /\left(2 \omega_{0}^{2} G^{3} m\right), S$ the surface of the plane electrodes at rest, $G$ the gap between the two electrodes, $m$ the mass of the resonator and $\varepsilon$ the permittivity of the material between the two electrodes.

In this case, (4) may be written:

$h\left(V, \omega, g, \omega_{0}, Q, \eta\right)=1-$

$\frac{V^{6}}{16 \eta^{2} \omega_{0}^{2} V_{0}^{2} g^{6}}\left[\left[\frac{\left(\omega_{0}^{2}-\omega^{2}\right) s^{g}(V)^{3}-2 \eta \omega_{0}^{2}}{d^{g}(V)}\right]^{2}+\frac{\omega^{2} \omega_{0}^{2} s^{g}(V)^{2}}{Q^{2}\left(1-s^{g}(V)\right)^{2}}\right]$

where $s^{g}(V)=\left(1-(V / g)^{2}\right)^{1 / 2}$ and $d^{g}(V)=\left(1-s^{g}(V)\right)+V^{2}\left(s^{g}(V)-2\right)^{2} / g^{2}$.

We will first focus on this model since it yields a first estimate of the parameters when the exact shape of the resonant mode is questionable. On the other hand, when this shape is well known, a more precise formula can be adopted, as illustrated on a clamped-clamped resonator in Subsection III.C.

\section{Electrostatic softening of a clamped-clamped beam resonator}

1) General expression

As the oscillation amplitude grows, a more realistic model than the plane-capacitance approximation must be considered. For instance, in the case of a clamped-clamped one-sided capacitive resonator, assuming $V_{0}=V_{a c t} / V_{b}<<1$, the dynamic behavior of the resonator is described by:

$\frac{d^{2} x}{d t^{2}}+\frac{\omega_{0}}{Q_{e}} \frac{d x}{d t}+\omega_{0}^{2} x=F_{c c}(V)$

with

$F_{c c}(V) \approx \xi_{c c} g^{1 / 2} \frac{(g+\kappa V \sin \omega t)\left(1+2 V_{0} \sin (\omega t+\varphi)\right)}{(g-V \sin \omega t)^{3 / 2}}$

where $\xi_{c c}=\eta \omega_{0}^{2} I_{0} / I_{1}, I_{0}=0.523, I_{l}=0.397, \kappa=0.014$ [12] and $\eta$ is defined in equation (6). 
For the sake of simplicity, we will assume $\kappa=0$ in the following equations. Finally,

$h\left(V, \omega, g, \omega_{0}, Q, \eta\right)=1-$

$\frac{V^{2}}{\alpha^{2} \omega_{0}^{2} V_{0}^{2} g^{2} l^{g}(V)^{2}}\left[\left[\omega_{0}^{2}-\omega^{2}-\frac{\alpha \omega_{0}^{2} g k^{g}(V)}{2 V}\right]^{2}+\frac{\omega^{2} \omega_{0}^{2}}{Q^{2}}\right]$ (9)

with $\alpha=2 \eta I_{0} / I_{1}$,

$$
\begin{aligned}
l^{g}(V)= & \frac{8(1+\kappa) V^{2}}{\pi g^{2} \sqrt{1+\frac{V}{g}}}\left[K-\left(1+\frac{V}{g}\right) E-\right. \\
& \left.\frac{8 \kappa g^{2}}{3 \pi V^{2}} \sqrt{1+\frac{V}{g}}\left(E-K\left(1-\frac{V}{g}\right)\right)\right] \\
k^{g}(V)= & \frac{4(1+\kappa) g}{\pi V\left(1-\frac{V}{g}\right) \sqrt{\frac{V}{g}}}\left[E-K\left(1-\frac{V}{g}\right)\right]- \\
& \frac{4 \kappa g}{\pi V \sqrt{1+\frac{V}{g}}}\left[K-E\left(1+\frac{V}{g}\right)\right]
\end{aligned}
$$

where $K$ (resp. $E$ ) stands for the complete elliptic integral of the first (resp. second) kind, with argument $2 V /(g+V)$.

We will work with this model since it is the best suited to our particular application.

\section{2) Simplified expression}

Taylor-series expansions of the electrostatic force are often reported in the literature $[13,14]$ since they yield simpler formulations than the exact expression of the electrostatic force. For instance, after a third-order expression of (8), $h$ is equal to:

$$
\begin{aligned}
& h\left(V, \omega, g, \Omega_{0}, Q_{e}, \gamma\right)= \\
& 1-\frac{35^{2} V^{2}}{32^{2} V_{0}^{2} \gamma^{2} g^{2} \Omega_{0}^{4}}\left[\left(\Omega_{0}^{2}-\omega^{2}-\frac{3}{4} \gamma \Omega_{0}^{2} \frac{V^{2}}{g^{2}}\right)^{2}+\frac{\omega^{2} \Omega_{0}^{2}}{Q_{e}^{2}}\right]=0
\end{aligned}
$$

where $\Omega_{0}^{2}=\frac{35 \omega_{0}^{2}}{35-24 \gamma}$ and $\gamma \Omega_{0}^{2}=-\frac{35 \varepsilon S V_{b}^{2} I_{0}}{32 m G^{3} I_{1}}$.

Even though this model is only valid for low-amplitude oscillations, it will be fitted to experimental results and compared to (7) and (9) in Section V.

\section{SimUlations}

The aim of this section is to explain the fitting procedure and illustrate its accuracy on simulated data. We illustrate our method on resonators described by (7) and (9).

\section{A. Formulation of the characterization problem}

Let us now consider a set of $N$ measurements $\left(V_{k}, \omega_{k}\right)$ with $k \in[1 ; \mathrm{N}]$, obtained by sweeping $\omega$ at a constant actuation amplitude. Our aim is to identify, with this data, the values of parameters $\omega_{0}, Q$ and $\theta$ that characterize the resonator. A general characterization method consists in minimizing:

$$
H\left(g, \omega_{0}, Q, \theta\right)=\sum_{k=1}^{N}\left[h\left(V_{k}, \omega_{k}, g, \omega_{0}, Q, \theta\right)\right]^{2}
$$

where $h$ is defined by (4). We fit a 4-parameter nonlinear frequency response via the minimization of (11).

\section{B. Characterization procedure}

In the following sections, we assume that the experimenter has a priori information on the values of $g, \omega_{0}, Q$ and $\eta$ within a range of $\pm 100 \%$. We run nonlinear least-squares fitting procedures based on the trust-region algorithm, with a lower bound $\left(g, \omega_{0}, Q, \eta\right)=(\max (V), 0,0,0)$ where $\max (V)$ corresponds to the maximal amplitude measured during the sweep. This last condition ensures that $A<1$. We apply the iterative procedure depicted in Fig. 1 with random initial conditions on the four parameters $\left(g, \omega_{0}, Q, \eta\right)$.

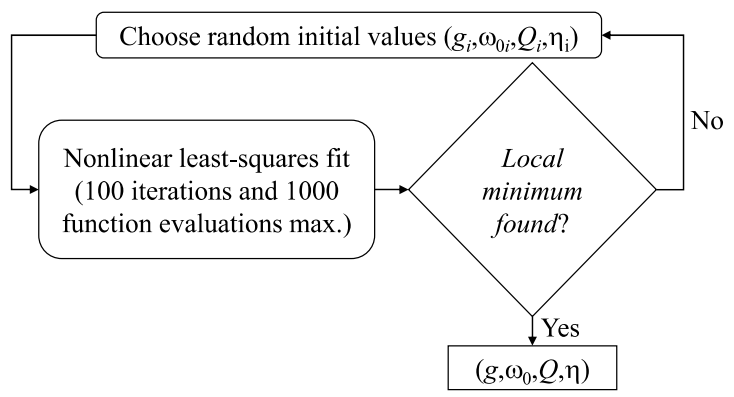

Fig. 1 - Flow-chart of the recursive procedure.

In Subsection III.B. and III.C., this procedure is illustrated on sets of simulated data corresponding to the planecapacitance model (7) and then the CC model (9). Each set of 40-point data stands for 40 experimental amplitude measurements where the actuation frequency is swept down around the peak response. We choose $g=1, f_{0}=\omega_{0} /(2 \pi)=68 \mathrm{kHz}$, $Q=10000, V_{a c t}=10 \mathrm{mV}, 25 \mathrm{mV}, 50 \mathrm{mV}$ and $70 \mathrm{mV}$ and $V_{b}=60 \mathrm{~V}$ since these parameters are realistic for our MEMS resonators [15]. We choose $\xi_{c c}=\xi_{p}\left(=1 \cdot 2 \cdot 10^{-9} \mathrm{~s}^{-2}\right)$ so that equivalent actuation forces are applied in both cases.

\section{Characterization of plane-capacitance MEMS resonator}

The results of the described procedure for a planecapacitance resonator are reported in Fig. 2 where the normalized frequency is defined as $\omega / \omega_{0}$. The crosses correspond to simulated results (i.e. pseudo-experimental results) and the continuous lines to the curves fitted to these responses according to the procedure listed in Fig. 1. 


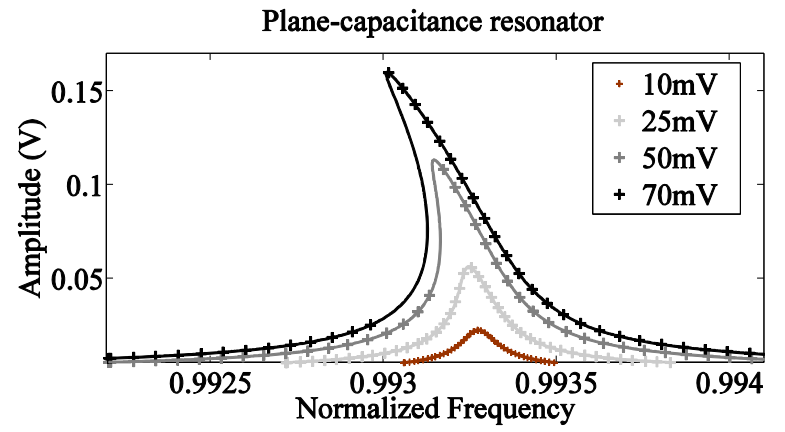

Fig. 2 - Typical frequency responses of a plane-capacitance resonator for different values of $V_{\text {act }}$. Simulated data sets (crosses) and corresponding nonlinear least-squares fits (solid lines).

Fig. 2 highlights the precision of the curve fittings. We checked that the four parameter $\left(f_{0}, Q, \eta, g\right)$ are determined without bias, to verify the accuracy of the presented method.

\section{Characterization of a clamped-clamped resonator}

Assuming a clamped-clamped resonator described by (9), Fig. 3 reports the results of the fitting procedure with the parameter and voltage values given in Subsection IV.A. The parameter extraction and curve fitting for the CC model is as accurate as for the parallel-plate model.

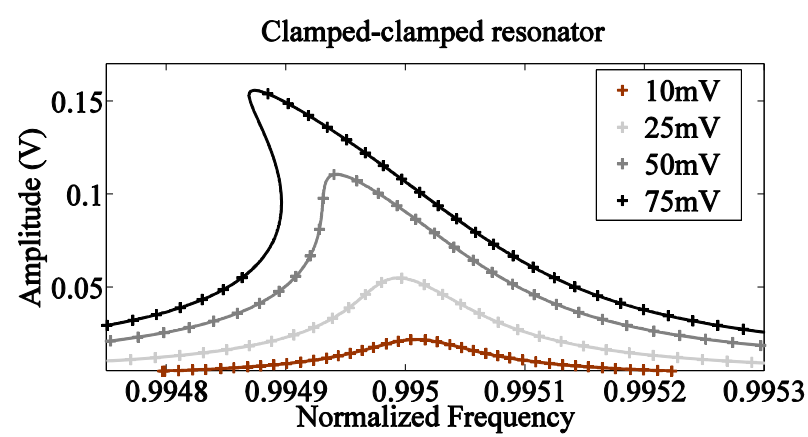

Fig. 3 - Typical frequency responses of a clamped-clamped resonator for different values of $V_{\text {act }}$. Simulated data sets (crosses) and corresponding nonlinear least-squares fits (continuous lines).

One may notice that our method is unaffected by the presence of hysteretic cycles. Moreover, it does not require bifurcation point measurements. Few measurements are required close to the peak amplitude and one single series of sweep-down measurements is sufficient to determine the four unknown parameters. In the next section, we will present our experimental setup and apply our method on experimental data.

\section{EXPERIMENTAL RESULTS}

\section{A. Experimental setup}

The resonator characterized in this study was originally developed by SEXTANT Avionics (currently THALES) [15]. It is industrially assembled by the fusion-bonding of three etched silicon wafers and consists of a resonant beam resting on a rectangular diaphragm. During the manufacturing process, the beam is encapsulated in vacuum to achieve a high mechanical Q-factor $\left(Q \approx 2 \times 10^{4}\right.$ at $V_{b}=15 \mathrm{~V}$ and $\left.V_{a c t}=10 \mathrm{mV}\right)$. The natural frequency $f_{0}=\omega_{0} / 2 \pi$ of the device is close to $68 \mathrm{kHz}\left(\omega_{0} \approx 4.3 \times 10^{5}\right.$ $\left.\operatorname{rad} . \mathrm{s}^{-1}\right)$.

In our setup, a bias voltage $V_{b}=60 \mathrm{~V}$ is applied to the resonator. It is directly actuated in open-loop by a waveform generator delivering voltage pulses of width $T_{p}=1 \mu \mathrm{s}$ and amplitude $V_{\mathrm{p}}=2.5 \mathrm{~V}$, repeated every $T$ seconds, where $T(=1 / f)$ is close to a multiple of $2 \pi / \omega_{0}$ (here $22 \pi / \omega_{0}$ ). In this setup, $V_{a c t}=V_{\mathrm{p}} T_{p} \omega_{0} /(11 \pi)$ with similar considerations as in [9]. This actuation scheme combined with adequate signal processing suppresses parasitic capacitance effects on the frequency response as explained in [9].

\section{B. Experimental results on the clamped-clamped resonator}

The following results are obtained within one series of measurements where the frequency is only swept down. Fig. 4 reports the experimental measurements and fitted curves after the non-linear least-squares fitting procedure explained in Section IV. In comparison to linear regimes presented in [9], the SNR of the presented measurements are up to 10 times better.

Knowing that the first resonant mode of the resonator is an out-of-plane clamped-clamped mode [16], we choose the model (9) for the curve fitting. We report in Fig. 4.a the fit obtained with the plane capacitance model (7), the CC model (9) and the TS model (10). As Fig.4.a. highlights, our method can be adapted to the three models. The fitted curves highlight a good agreement between the three models and experimental results (see Fig. 3). According to the curve fitting, the maximal oscillation amplitude achieved in this regime is close to $24 \%$ of the gap distance.

In the next subsections, we compare the results of the three models to estimate the robustness of our method against noise and modeling errors.

\section{Accuracy of the curve fitting}

In order to evaluate the quality of the experimental curve fitting, we compare the predicted upper bifurcation point with the experimental jump point measured with the frequency sweep-down. In a perfect scenario, the frequency of the upper bifurcation point corresponds to the experimental jump discontinuity, represented by the dashed dark line in Fig. 4. 


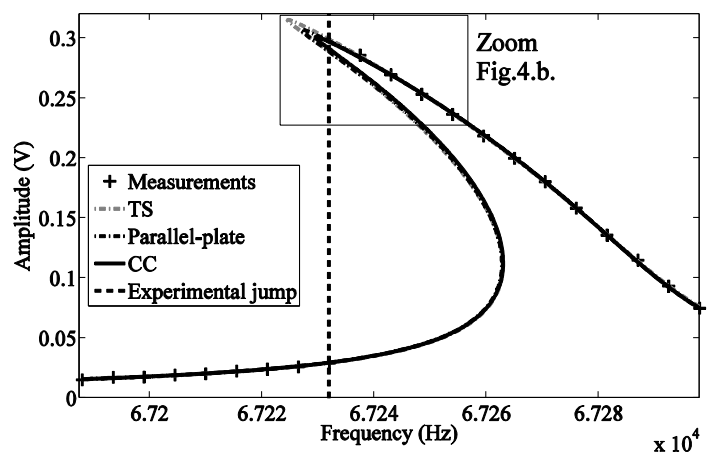

Fig. 4.a. - Experimental frequency response: Measurement points (crosses) and corresponding nonlinear least-squares fits. The dash-dotted dark line corresponds to (7), the solid dark line to (9), and the dash-dotted grey line to (10). The experimental bifurcation frequency is represented by the dashed dark line.

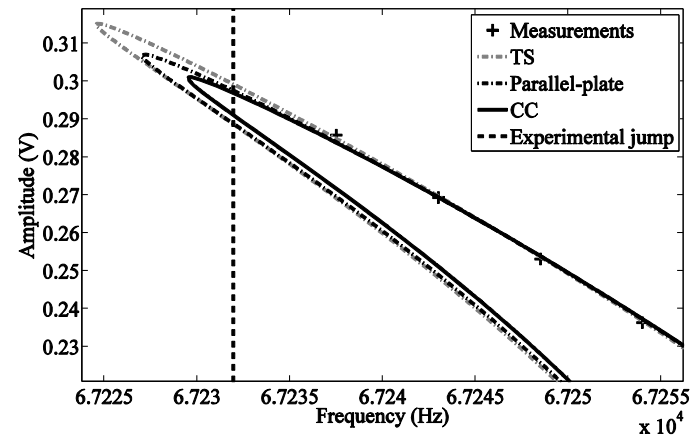

Fig. 4.b. - Zoom on the upper bifurcation point.

The upper bifurcation point predicted by the clampedclamped model fits better than the one predicted by the Taylor series and the plane-capacitance models, justifying the need for the model described by (9). However, the three models predict the position of the jump discontinuity quite well. The maximal error on the upper bifurcation frequency is close to $0.01 \%$ (TS model). In Subsection V.D., we will compare the parameters extracted with the different models to the clamped-clamped model (9).

\section{Robustness against modeling errors}

Considering that the clamped-clamped model (9) is the most accurate, we report in Table I the relative error on the parameters extracted with the different models.

Table I. Relative error on the estimated parameters with the $\mathrm{CC}$ model as a reference $\left(\beta=\varepsilon S /\left(m G^{3}\right)\right)$.

\begin{tabular}{|l|c|c|c|c|}
\hline Model & $\omega_{0}$ & $Q$ & $\beta$ & $g$ \\
\hline TS & $0.03 \%$ & $7 \%$ & $2.8 \%$ & $-2.3 \%$ \\
\hline Parallel-plate & $0.1 \%$ & $1.9 \%$ & $8.7 \%$ & $21 \%$ \\
\hline
\end{tabular}

Table I illustrates the robustness of our method against modeling errors. All the models yield a good estimation of the natural frequency and the Q-factor in large-amplitude oscillation regimes.
However, the parallel-plate model overestimates $\beta\left(=\varepsilon S /\left(m G^{3}\right)\right)$ and $g$. These overestimations may be partially explained by the influence of the static displacement of the resonator. Our model may be adjusted to take into account this static displacement in (4). Second-order corrections may also be related to the nonlinear detection, which means that, instead of $V=g A$, one should use $V=g \zeta(A) A$ where the function $\zeta$ depends on the considered model.

\section{CONCLUSION}

In this paper, we developed a generic characterization method and applied it to capacitive MEMS resonators. We validated the method on simulated and experimental data illustrating its accuracy in large amplitude regimes. The general formulation of MEMS characterization problems was adapted to several models corresponding to common approximations. For all the presented models, this general formulation yields unbiased parameter estimation without requiring precise peak amplitude or bifurcation point measurements. Hence, capacitive MEMS devices can be characterized in nonlinear regime with one single series of measurements. In a near future, nonlinear regimes may be exploited to study the amplitudedependence of quality factors of MEMS resonators subject to nonlinear damping. Since our sensor exhibits parametric resonance in non-linear regime [16], future work will also aim at experimental characterization of parametric resonance in open-loop regimes.

\section{REFERENCES}

[1] S. S. Rao, "Mechanical Vibrations", 3rd ed. Reading, MA: AddisonWesley, 1995, p. 204

[2] Z. Zeng, M.A.P. Pertijs and D. M. Karabacak, "An energy-efficient readout circuit for resonant sensors based on ring-down measurements", Review of Scientific Instruments, vol. 84, 2013.

[3] W. O. Davis, "Measuring Quality Factor From a Nonlinear Frequency Response With Jump Discontinuities", J. of Microelectromechanical systems, vol. 20, pp. 968-975, 2011.

[4] A. J. Dick, B. Balachandran, D. L. DeVoe and C. D. Mote Jr, "Parametric identification of piezoelectric microscale resonators", Sensors and Actuators A., vol. 16, pp. 1593 - 1601, 2006.

[5] J. E.-Y. Lee and A.A. Seshia, "Direct parameter extraction in feedthrough-embedded capacitive MEMS resonators", J. Micromech. Microeng., vol. 167, pp. 237-244, 2011.

[6] G. Vidal, F. Torres, N. Barniol, O. Gottlieb, "The influence of the parasitic current on the nonlinear electrical response of capacitive sensed cantilever beams", Proceedings of DTIP, pp. 255-260, 2014.

[7] A. T-H. Lin, J. E-Y. Lee, et al., "Enhanced transduction methods for electrostatically driven MEMS resonators", Solid-State Sensors, Actuators and Microsystems Conference (TRANSDUCERS) (2009), 561 - 564.

[8] V.J. Logeeswaran, F.E.H. Tay, et al, "First Harmonic (2f) Characterisation of Resonant Frequency and Q-Factor of Micromechanical Transducers", Analog Integrated Circuits and Signal Processing, 37 (2003), 17 33.

[9] A. Brenes, J. Juillard, F. Vinci Dos Santos and A. Bonnoit, "Characterization of MEMS resonators via feedthrough de-embedding of harmonic and subharmonic pulsed-mode response", Sensors and Actuators A, "in press".

[10] A. Brenes, J. Juillard, L. Bourgois and F. Vinci Dos Santos, "Parameter estimation from nonlinear frequency response of MEMS resonators", Proceedings of ISCAS, 2015, "in press". 
[11] R. Lifshitz and M. C. Cross, "Nonlinear Dynamics of Nanomechanical and Micromechanical Resonators", Review of Nonlinear Dynamics and Complexity, NJ: Wiley, 2008, p. 2-17

[12] J. Juillard, G. Arndt and E. Colinet, "Modeling of Micromachined Beams Subject to Nonlinear Restoring or Damping Forces", J. of Microelectromechanical systems, vol. 20, pp. 165-177, 2011.

[13] J. F. Rhoads et al., "Generalized parametric resonance in electrostatically actuated microelectromechanical oscillators", Journal of Sound and Vibration, vol. 296, pp. 797-829, 2006
[14] J. Juillard, "A comparative study of reduced-order modeling techniques for nonlinear MEMS beams", Proceedings of DTIP, pp. 261-265, 2014.

[15] J. Mandle, O. Lefort and A. Migeon, "A new micromachined silicon high-accuracy pressure sensor", Sensors and Actuators A, vol. 46-47, pp. 129 $132,1995$.

[16] A. Brenes, J. Juillard and F. Vinci Dos Santos., "Electrostaticallyinduced modal crosstalk phenomena in resonant MEMS sensors", Proceedings of DTIP, pp. 294-297, 2014. 